\title{
Does the neutron star in Her X-1 really show free precession?
}

\author{
R. Staubert; D. Klochkov, D. Vasco \\ Institut für Astronomie und Astrophysik, Universität Tübingen, Tübingen, Germany \\ E-mail: staubert (klochkov, vasco) dastro.uni-tuebingen.de
}

\section{K. Postnov, N. Shakura}

Sternberg Astronomical Institute, Lomonossov University, Moscow, Russia

E-mail: kpostnov@gmail.com, nikolai.shakura@gmail.com

\section{R. Rothschild}

Center for Astrophysics and Space Sciences, Univ. of California San Diego, San Diego, USA

E-mail: rrothschildeucsd.edu

\section{J. Wilms}

Dr. Remeis-Sternwarte Bamberg and Erlangen Center for Astroparticle Physics, Universität Erlangen-Nürnberg, Germany

E-mail: wilms@sternwarte.uni-erlangen.de

The accreting X-ray pulsar Her X-1 shows two types of long-term variations, both with a period of $\sim 35 \mathrm{~d}$ : 1) A modulation of the flux with a ten day long Main-On and a $5 \mathrm{~d}$ long Short-On, separated by two Off-states, and 2) A systematic variation of the shape of the $1.24 \mathrm{~s}$ pulse profile. While there is general consensus that the flux modulation is due to variable shading of the X-ray emitting regions on the surface of the neutron star by the precessing accretion disk, the physical reason for the variation of the pulse profiles had remained controversial. Following the suggestion by Trümper et al. (1986) that free precession of the neutron star may be responsible for the variation of the pulse profiles, we had developed physical models of strong feedback interaction between the neutron star and the accretion disk in order to explain the seemingly identical values for the periods of the two types of variations. In a deep analysis of pulse profiles observed by several different satellites over the last three decades we find now that the clock behind the pulse profile variations shows exactly the same erratic behavior as the turn-on clock, even on short time scales $(\sim 100 \mathrm{~d})$, suggesting that there may in fact be only one $35 \mathrm{~d}$ clock in the system. If this is true, it presents a serious challenge for the idea of free precession of the neutron star and calls for alternative physical models for the variation in pulse shape.

8th INTEGRAL Workshop "The Restless Gamma-ray Universe" - Integral2010, September 27-30, 2010, Dublin, Ireland

\footnotetext{
*Speaker.

†presented by D. Klochkov on behalf of R. Staubert et al.
} 


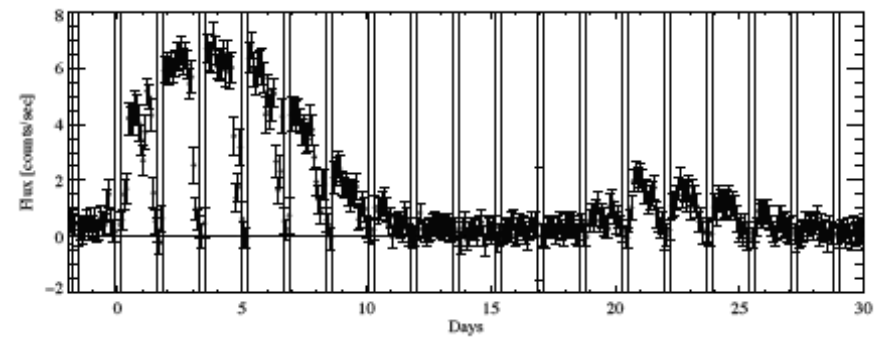

Figure 1: Average $35 \mathrm{~d}$ flux profile of Her X-1, generated by accumulating light curves observed by RXTE/ASM for $35 \mathrm{~d}$ turn-ons around binary phase 0.2 (Fig. 3 of Klochkov et al. 2006). The vertical lines indicate the binary eclipses.

\section{1. $35 \mathrm{~d}$ flux modulation and turn-on history}

Her X-1 shows modulation of its X-ray flux on a $\sim 35 \mathrm{~d}$ period, with a "Main-On" and a "Short-On", separated by two "Off" states. The sharp increase of the flux defines the beginning of a cycle and is called "turn-on", it generally occurs either around binary phases 0.2 or 0.7. Fig. 1 shows the mean flux profile for phase 0.2 turn-ons as observed by RXTE/ASM (Klochkov et al., 2006). The 35 day modulation of the X-ray flux is generally explained by the precession of the accretion disk, that regularly blocks the view to the $\mathrm{X}$-ray emitting regions near the magnetic poles of the neutron star.

The $35 \mathrm{~d}$ turn-on clock is quite irregular, allowing the length of an individual cycle to be either $20.0 \times \mathrm{P}_{\text {orb }}, 20.5 \times \mathrm{P}_{\text {orb }}$, or $21.0 \times \mathrm{P}_{\text {orb }}$ (Staubert et al., 2006) (with a small fraction of cases showing longer or shorter cycles). Adopting $\mathrm{P}_{35}=20.5 \times \mathrm{P}_{\text {orb }}(=34.85 \mathrm{~d})$ as the mean ephemeris period, the turn-on history can be described by the so called $(O-C)$-diagram, which plots the difference between the observed turn-on and the calculated turn-on (using this ephemeris period) as a function of cycle number (or time). Fig. 2 shows $(O-C)$ since the discovery of Her X-1 until today as a function of time in MJD. The two versions of the diagram (left and right) differ in the number of $35 \mathrm{~d}$ cycles assumed to have occurred during the 602 day long Anomalous Low (AL3), which is centered at $\sim$ MJD 51500. During this gap, the X-ray flux was very low because of the blocking by the low inclination accretion disk. For the interpretation of Fig. 2 we refer to Staubert et al. (2009), in the following called the "Two-clocks paper". In Fig. 2-left the gap corresponds to 18 cycles with a (short) mean duration of $19.7 \times \mathrm{P}_{\text {orb}}$, which is probably the correct physical interpretation for a continued precession of the accretion disk. In Fig. 2-right the gap corresponds to 17 cycles with a (long) mean duration of $20.8 \times \mathrm{P}_{\text {orb }}$, which can be associated with a semi-regular clock with a mean period of $20.8 \times \mathrm{P}_{\text {orb }}=34.88 \mathrm{~d}$. In the "Two-clocks paper" (Staubert et al., 2009) this period was associated with an underlying clock, viewed to be rather regular, namely (free) precession of the neutron star, assumed to be responsible for the observed periodic variation of the shape of the pulse profiles. In this model, it was assumed, that the precession of the neutron star is the master clock and that the precession of the accretion disk is locked to that of the neutron star by evidently existing strong physical feed-back in the system, (nearly) synchronizing the periods of the "two clocks". 

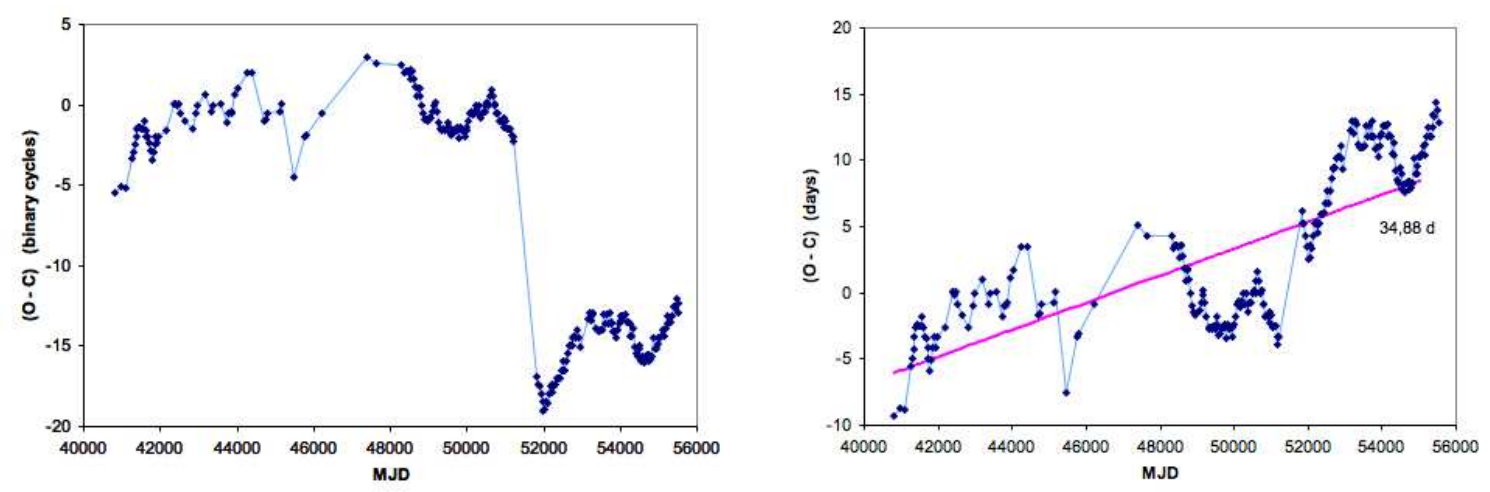

Figure 2: Turn-on history of Her X-1: the $(O-C)$-diagram. Left: assuming that during the Anomalous Low around MJD 51500 (AL3) there were eighteen 35 d cycles. Right: the same assuming that there were only seventeen $35 \mathrm{~d}$ cycles (see Staubert et al., 2009 and text for further discussion).

\section{Pulse profile variations}

In observations of Her X-1 by EXOSAT in 1984, Trümper et al. (1986) discovered that the $1.24 \mathrm{~s} X$-ray pulse profiles vary in shape as a function of the phase of the $35 \mathrm{~d}$ flux modulation. Observations by Ginga in 1989 and by RXTE starting in 1996 confirmed these findings and have added a wealth of detailed information on the combined pulse shape and spectral evolution of the pulsars beamed emission (e.g., Scott et al., 2000). Trümper et al. (1986) had suggested that the systematic variations in pulse shape is due to free precession of the neutron star: the viewing angle towards the X-ray emitting polar cap region of the neutron star varies with the phase of the neutron star precession. Shakura et al. (1998) applied a model of a precessing triaxial model to pulse profiles of Her X-1 observed by HEAO-1, Ketsaris et al. (2000) did so for profiles observed by RXTE/PCA. Using all observations by RXTE from 1996 until 2005 we have verified that the shape of the pulse profiles is reproduced every $\sim 35$ days. A careful timing analysis was performed of all archived RXTE data on Her X-1 and pulse profiles were generated by folding with the measured pulse periods. As an example, Fig. 3-left shows a set of pulse profiles (PCA, 9-13 keV) for eight different $35 \mathrm{~d}$ phases. The variation of the pulse shape is evident. This systematic analysis has lead to the development of the "Two-Clocks-Model" (Staubert et al., 2009) and the successful modeling of the observed pulse profiles by a model of point- and ring-like emission from the polar caps of a neutron star with an offset-dipole field under the assumption of free precession (Postnov, 2004).

We have then started a model-independent investigation of the periodic pulse profile variations by constructing a template of those variations for the Her X-1 "Main-On" for photon energies 9$13 \mathrm{keV}$. A set of observations were selected, providing a good coverage of the $35 \mathrm{~d}$ phase range -0.05 to 0.15 . This template contains flux normalized pulse profiles for every 0.01 in phase. Any 9-13 keV pulse profile observed during a Main-On can then be compared to this template and the $35 \mathrm{~d}$ phase can be determined (by $\chi_{\min }^{2}$ minimization). For the RXTE data we find that this is generally possible to an accuracy of \pm 0.02 in phase. First results of using this template with profiles observed by Ginga, RXTE and INTEGRAL were presented by Staubert et al. (2010), showing that the turn-on history and the history of variations of the pulse profiles appeared to be 

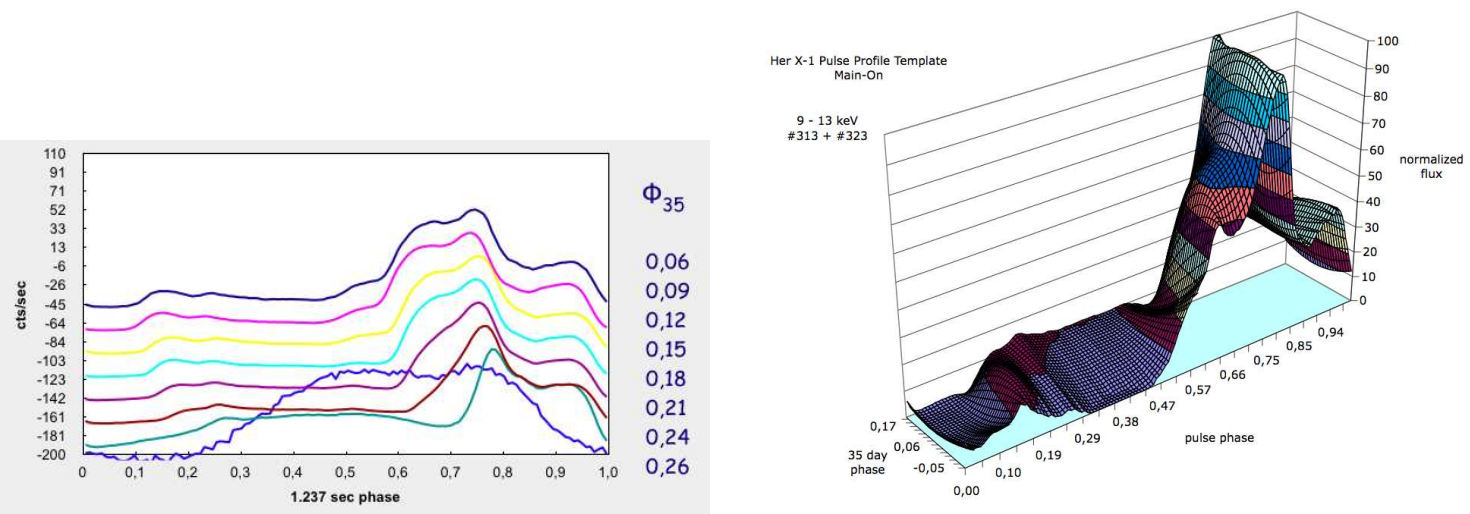

Figure 3: Left: Pulse profiles of Her X-1 of 35 day cycles of Dec 01 (cycle no. 313) and Nov 02 (cycle no. 323) as a function of 35 day phase (RXTE, 9-13 keV). Right: 3D pulse profile template for the Her X-1 Main-On ( $35 \mathrm{~d}$ phase -0.05 to 0.22 ) of cycles 313 and 323 in the energy range $9-13 \mathrm{keV}$. The resolution is 128 bins in pulse phase and 28 bins in $35 \mathrm{~d}$ phase.

strictly parallel, implying that the neutron star precession (if it is indeed the reason behind the pulse profile variations) is synchronized to the precession of the accretion disk. This raised the question, how the neutron star would be able to significantly change its precessional period on rather short time scales.

We have since refined this method by constructing an extended template from $R X T E / \mathrm{PCA}$ observations of two $35 \mathrm{~d}$ cycles (nos. 313 and 323), which together provide a uniform coverage of a complete "Main-On" in the $35 \mathrm{~d}$ phase range -0.05 to 0.22 , again with a resolution of 0.01 . The details of the construction of this template, its characteristics, and its usage are described by Staubert et al. (2011) (this Volume). A 3D-representation of the template (being a matrix of 128 bins in pulse phase by 28 bins in $35 \mathrm{~d}$ phase) is given in Fig. 3-right.

\section{Results and discussion}

We compare the characteristics of the two $35 \mathrm{~d}$ modulations (of the flux and of the variation in pulse profiles) by plotting both into an $(O-C)$-diagram. For the flux, we use the turn-on, also called the "accretion disk phase-zero" and plot the $(O-C)$ as in Fig. 2-right. Correspondingly, we determine a "pulse profile phase-zero" for all the Main-Ons for which there is at least one observed pulse profile (for most Main-Ons there are several profiles). The "pulse profile phase-zero" is defined and calibrated using those cycles which were used to construct the template: for cycles 313 and 323 both times of "phase-zero" are identical (by definition). For any other cycle "pulse profile phase-zero" is found by determining the $35 \mathrm{~d}$ phases of all available profiles by comparison with the template and a subsequent linear extrapolation to phase zero using the fixed period of $34.85 \mathrm{~d}$ (see Fig. 5-left of Staubert et al., 2011). Using pulse profiles of other Main-On cycles of Her X-1 observed by RXTE over the last three decades plus one from Ginga and one from INTEGRAL, we find that these systematic variations are very stable and reproducible. The constructed template therefore allows to determine the $35 \mathrm{~d}$ phase for any observed Main-On pulse profile in the 9$13 \mathrm{keV}$ range. 


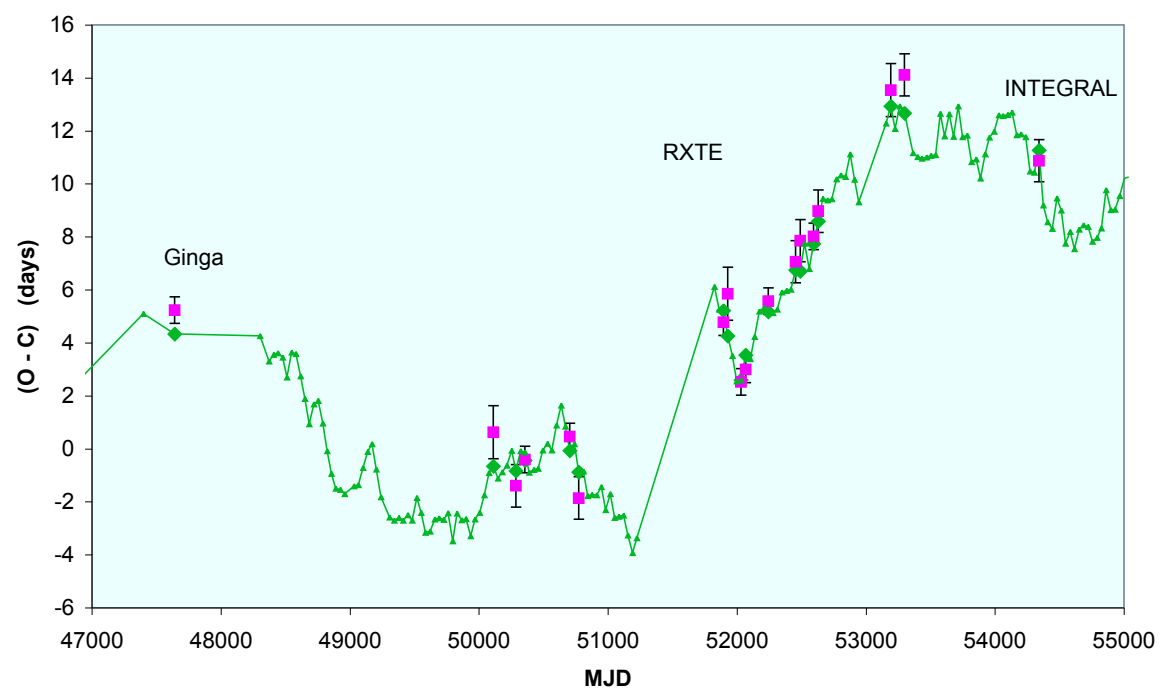

Figure 4: $(O-C)$ values in units of days for observed turn-ons (green) and for so far generated "pulse profile phase-zero" values from pulse profile fitting (mangenta), using profiles 9-13 keV profiles for several Main-Ons observed by RXTE, plus one from Ginga and one from INTEGRAL.

The observational result in the form of an $(O-C)$-diagram is summarized in Fig. 4. The green points (connected by the solid green line) represent the observed turn-on times (equal to "accretion disk phase-zero"). The magenta points represent the times of "pulse profile phase-zero" as determined from the comparison of observed pulse profiles with the pulse profile template and subsequent extrapolation to phase zero. The observational evidence is quite clear: within statistical uncertainties, the values for phase-zero as determined by the two different methods are identical, and the "pulse profile clock" is just as irregular as the "turn-on clock". Both clocks appear to be perfectly synchronized. We emphasize here, that the above result is completely model independent, it is obtained using observational data only. However, the latest results from the continued effort in modeling the observed pulse profiles by a model assuming free precession of the neutron star (Postnov, 2004; a publication is in preparation) leads to the same conclusion. This does suggest abandoning the concept of "two clocks" and to assume the existence of just one underlying clock which controls the variations of both zero-phases: that of the turn-ons, and that of pulse profile phase zero.

What does the above result mean for the concept of (free) precession of the neutron star in Her X-1? We distinguish between two assumptions.

First, assuming the precession of the neutron star does indeed exist and is responsible for the variation of the pulse profiles and for those of the turn-on times, we would then have to explain, how the neutron star could change its precessional period by several percent on rather short time scales ( $\sim 100 \mathrm{~d})$. There seem to be no external forces that are strong enough to change the precessional period, e.g. by applying a torque to the principle axis of inertia. The only possible origin could be inside the neutron star, that is, if glitches occur or if the complex physics of the interior of a highly magnetized neutron star with its crust and liquid core did allow for time variable phenomena. For the relevance of free precession to our understanding of matter at supra-nuclear densities, see e.g., 
Link (2007). Equally difficult is the explanation of how the neutron star, if it is indeed the one master $35 \mathrm{~d}$ clock in the system, would be able to transmit its precessional motion to that one of the accretion disk (assuming that our long-term concept is correct, that the turn-ons are due to the precession of the accretion disk). We do indeed see strong feed-back in the binary system, which has lead Staubert et al. (2009) to assume that the precession of the neutron star may be the master clock in the system, under the assumption, however, that the neutron star clock would be rather stable and that the accretion disk would have "a life of its own" providing the freedom to deviate from the strict clocking of the master - as observed in the turn-on history. We now see, that the turn-ons and the pulse profiles vary in strict synchronization, requiring extremely strong feed-back, the existence of which appears questionable. Certainly, any neutron star precession would be far from "free".

Second, assuming that there is no neutron star precession, we would need a different explanation for the variation of the pulse profiles in synchronization with the turn-ons. We would have to assume that the precessional movement of the outer edge of the accretion disk, thought to be responsible for the $35 \mathrm{~d}$ flux modulation, is mirrored at the inner edge of the accretion disk, and that its interaction with the magnetosphere of the neutron star and the way in which the accretion of matter proceeds along the field lines of the (probably) multi-pole magnetic field, is varying with precessional phase. Scott et al. (2000), on the basis of Ginga and early RXTE observations, proposed a model in which the changes in the shape and spectral appearance of the pulse profiles are qualitatively explained by a combination of occultation of the X-ray emitting regions by a tilted and twisted precessing inner accretion disk and changes in the accretion geometry by the changing relative orientation between disk and neutron star magnetosphere. A detailed quantitative model would be needed to verify this idea for the generation of the multi-component pulse profiles.

\section{References}

[1] Ketsaris N. A., Kuster M., Postnov K. A., Shakura N. I., Staubert R., Willms J. 2000, in Proc. "Hot Points in Astrophysics", Joint. Inst. for Nucl Res., Dubna, p.192

[2] Klochkov D., Shakura N. I., Postnov K. A., Staubert R., Willms J., Ketsaris N. A. 2006, Astron. Lett. 32, 804

[3] Link B. 2007, Astroph.\&Space Sc., 308, 435

[4] Postnov K. 2004, http://astro.uni-tuebingen.de/staubert65/talks/postnov.pdf

[5] Scott D. M., Leahy D., Wilson R.B. 2000, ApJ, 539, 392

[6] Shakura N. I., Postnov K., Prokhorov M. 1998, A\&A, 331, L37

[7] Staubert R., Schandl S., Klochkov D., Wilms J., Postnov K., Shakura N. 2006, in AIP Conf. Proc., Vol. 840, 65

[8] Staubert R., Klochkov D., Postnov K., Shakura N. I., Willms J., Rothschild R. E. 2009, A\&A 494, 1025

[9] Staubert R., Klochkov D., Postnov K., Shakura N., Wilms J., Rothschild R. E. 2010, in AIP Conf. Proc., Vol. 1248, 209 (arXiv:0911.3566)

[10] Staubert R., Vasco, D., Klochkov D., Wilms J. 2011, in Proceed. 8th INTEGRAL Workshop "The Restless Gamma-ray Universe", Dublin, 2010, this Volume

[11] Trümper J., Kahabka P., Oegelmann, H., Pietsch W., Voges W. 1986, ApJ 300, L63 